\title{
Efeitos dos intervalos de tempo de apresentação de conhecimento de resultados (CR) na aquisição de habilidades motoras
}

CDD. 20.ed. 152.3

\author{
Márcio Mário VIEIRA*/** \\ Herbert UGRINOWITSCH* \\ Guilherme Menezes LAGE ${ }^{* / * * *}$ \\ Rodolfo Novellino BENDA*
}

*Universidade Federal de Minas Gerais.

**Faculdade Estácio de

Sá de Belo Horizonte.

***Universidade FUMEC.

\begin{abstract}
Resumo
0 aspecto temporal do Conhecimento de Resultados (CR) é composto por três intervalos: pré-CR, pós-CR e o intervalo intertentativas. Um problema sobre este tópico é que a manipulação de um intervalo implica na alteração dos outros, o que sugere uma análise conjunta desses intervalos. 0 presente estudo investigou os efeitos dos intervalos de tempo de apresentação de CR na aquisição de habilidades motoras. Foi utilizada uma tarefa de posicionamento manual, que consistia em transportar três bolas de tênis entre seis recipientes em ordem e tempo alvo pré-estipulados. 0 instrumento continha uma plataforma com seis recipientes e uma central de controle ligada ao microcomputador. 0 estudo teve a fase de aquisição, com 30 tentativas com a sequência (4-2/5-3/6-1) e tempo alvo (3000 ms) definidos pelo experimentador. Dez minutos após o término da fase de aquisição foi aplicado o teste de transferência imediata e, após 24 horas, o teste de transferência atrasada. Ambos os testes tiveram de 15 tentativas de prática, de uma nova sequência de movimento (6-1/5-3/4-2) e tempo alvo (4000 ms), sem fornecimento de CR. Noventa universitários foram divididos em nove grupos ( $\mathrm{n}$ = 10): três grupos com quatro segundos, três grupos com oito segundos e três grupos com 16 segundos de intervalo intertentativas, todos com intervalos pré e pós-CR distintos. Os resultados mostraram que menores intervalos intertentativas tiveram melhor desempenho nos testes. Isto sugere que o tempo para processamento de informação não deve ser longo, o que aumentaria a demanda de atenção e a exigência de memória.
\end{abstract}

UnITERMOS: Feedback; Conhecimento de resultados; Intervalo de atraso; Intervalo intertentativas.

\section{Introdução}

Os intervalos de tempos de apresentação de conhecimento de resultados (CR) são reconhecidos como variáveis relacionadas ao momento de fornecimento de "feedback" bem como de elaboração da estratégia para as novas tentativas (GODINHO \& MENDES, 1996). A manipulação dos aspectos temporais do CR tem como objetivo conhecer seu efeito no processo de aprendizagem, nomeadamente quais as operaçóes e mecanismos são facilitados ou perturbados pela sua presença ou ausência, como também pela variação da extensão do tempo disponível (GODINHO \& MENDES, 1996; Travlos \& Pratt, 1995).

Os tempos de apresentação do CR referem-se habitualmente a três variáveis: intervalo pré-Conhecimento de Resultados (pré-CR), intervalo pós-Conhecimento de Resultados (pós-CR) e o intervalo intertentativas.
O intervalo pré-CR consiste na extensão de tempo entre o término da tentativa e o recebimento do CR (SCHмidT, 1988). Esse intervalo é utilizado para que o indivíduo possa estimar seu próprio erro (SWINNEN, 1996; Travlos \& Pratt, 1995). Após o término da execução até o momento que é fornecido o $\mathrm{CR}$, o aprendiz dispóe de informações sensoriais do movimento desempenhado. Nesse intervalo é possível realizar a operação de análise do "feedback" intrínseco (ADAMS, 1971; SCHMIDT, 1975), fortalecendo assim os mecanismos de detecção e correção de erros (SWINNEN, SCHMIDT, NichOLSON \& SHAPIRO, 1990).

A investigação desta temática mostrou que intervalos mais curtos, contudo não próximos a zero, apresentaram melhores resultados que intervalos de oito segundos (SWINNEN et al., 1990). Resultados 
similares concluíram que o intervalo de atraso de CR é importante para a aprendizagem motora, em tempo suficiente para organizar estratégias, além do processamento da informação por meio dos mecanismos de detecção do erro via "feedback" intrínseco (CARNAHAN, Hall \& Lee, 1996). Por outro lado, estudos que utilizaram tarefas complexas, como o arremesso (Liu \& WrisberG, 1997) ou a "tacada" do golfe (Liu, GervaIs \& CROSTON, 2001), não encontraram resultados favoráveis aos intervalos pré-CR mais curtos. Tais achados levaram a assumir que a extensão do intervalo pode estar relacionada às características da tarefa, como por exemplo, a sua complexidade (Palhares, Lage, VieIra, UGRINOWITSCH \& BENDA, 2006).

$\mathrm{O}$ intervalo pós-CR, intervalo entre o recebimento do CR e a próxima tentativa, corresponde ao período de tempo no qual são executadas comparações entre o "feedback" intrínseco e o CR, e como consequência, são elaboradas as estratégias para a tentativa seguinte (GAllagher \& ThOMAS, 1980; SCHMidT, 1988). Tal intervalo tem sido considerado determinante para a aquisição de habilidades motoras (Ramella, 1983; Salmoni, Schmidt \& Walter, 1984) e resultados encontrados confirmam esta importância (GALlagher \& ThOMAS, 1980; Magill, 1988; Ramella, 1983; Travlos, 1999; Travlos \& Pratt, 1995; Weinberg, Guy \& Tupper, 1964).

Um problema nos estudos que investigaram esta temática é a extensão do intervalo. Esta posição tem suporte na falta de consenso sobre o efeito do intervalo pós-CR, pois um aumento na extensão do intervalo pós-CR no desempenho e aprendizagem são predominantemente nulos (MAGILL, 1977). Isto acontece provavelmente porque intervalos mais longos podem promover esquecimento da tarefa, o que sugere um ponto intermediário na extensão desses intervalos

\section{Métodos}

\section{Amostra}

Participaram do estudo 90 universitários voluntários, sendo 54 do sexo feminino e 36 do sexo masculino, com faixa etária entre 18 e 35 anos (idade média de 23,19+3,18 anos), inexperientes na tarefa e com consentimento livre e esclarecido. O estudo foi aprovado pelo Comitê de Ética em Pesquisa da Universidade, sob parecer de número ETIC 297/05.
(Vieira, Ennes, Lage, Palhares, Ugrinowitsch $\&$ BENDA, 2006). Outros estudos realizados com o intervalo pós-CR não encontraram efeito positivo do intervalo pós-CR, talvez por influência dos intervalos pré-CR e intertentativas, ou mesmo por características das tarefas utilizadas (BECKER, 2000; BECKER, MUSSINA \& Pearsons, 1963; Godinho, 1992; Godinho \& Mendes, 1996; Magill, 1994; Vieira et al., 2006).

$\mathrm{O}$ intervalo intertentativas corresponde ao período de tempo entre o término de uma tentativa e o início de outra, separando uma execução da execução posterior (SCHMidT, 1988). São poucos os estudos específicos sobre o efeito dessa variável no processo de aprendizagem, além de outros inferirem indiretamente seu papel, por meio da manipulação dos intervalos pré-CR e pós-CR (Godinho \& Mendes, 1996). Os estudos sobre essa variável se caracterizam por apresentarem apenas fase de aquisição, não sendo assim, possível analisar a aprendizagem (BILODEAU \& BilodeaU, 1958; Koegel, Dunlap \& Dyer, 1980; Simmons \& Snyder, 1983; Travlos \& Pratt, 1995).

Considerando que ao estudar o intervalo intertentativas manipula-se o intervalo pré-CR ou pós-CR, não há como se investigar tais intervalos sem uma análise conjunta. Não foi encontrado na literatura um estudo que envolvesse simultaneamente os três intervalos de tempo de apresentação de CR. Se este problema metodológico tem sido apontado como uma das limitaçóes para estudo deste tema (GodINHO \& MENDES, 1996), o resgate de tal questão com vistas a apresentar novas alternativas de investigação pode ser relevante para tal campo de conhecimento. Assim, o objetivo do presente estudo foi investigar os efeitos dos intervalos de tempo de apresentação de CR na aquisição de habilidades motoras.

\section{Instrumento e tarefa}

Foi utilizado um aparelho de posicionamento manual composto de duas estruturas: uma plataforma contendo seis recipientes enumerados de 1 a 6 e uma central de controle ligada a um microcomputador, constituída por diodos que fornecem estímulo visual para iniciar a tarefa e uma chave de resposta para registro das medidas de tempo (FIGURA 1). Um "software" foi desenvolvido para medida e armazenamento dos tempos fornecidos pelo aparelho. 
A tarefa utilizada consistiu no transporte de três bolas de tênis entre os recipientes mais próximos (números 4, 5 e 6) para os recipientes mais distantes da plataforma (números 1, 2 e 3) como nos estudos de Palmares et al. (2006). Ainda, foi utilizado um cronômetro da marca Diplomat com precisão em décimos de segundos para aferição dos intervalos de tempo de pré-CR e de pós-CR.

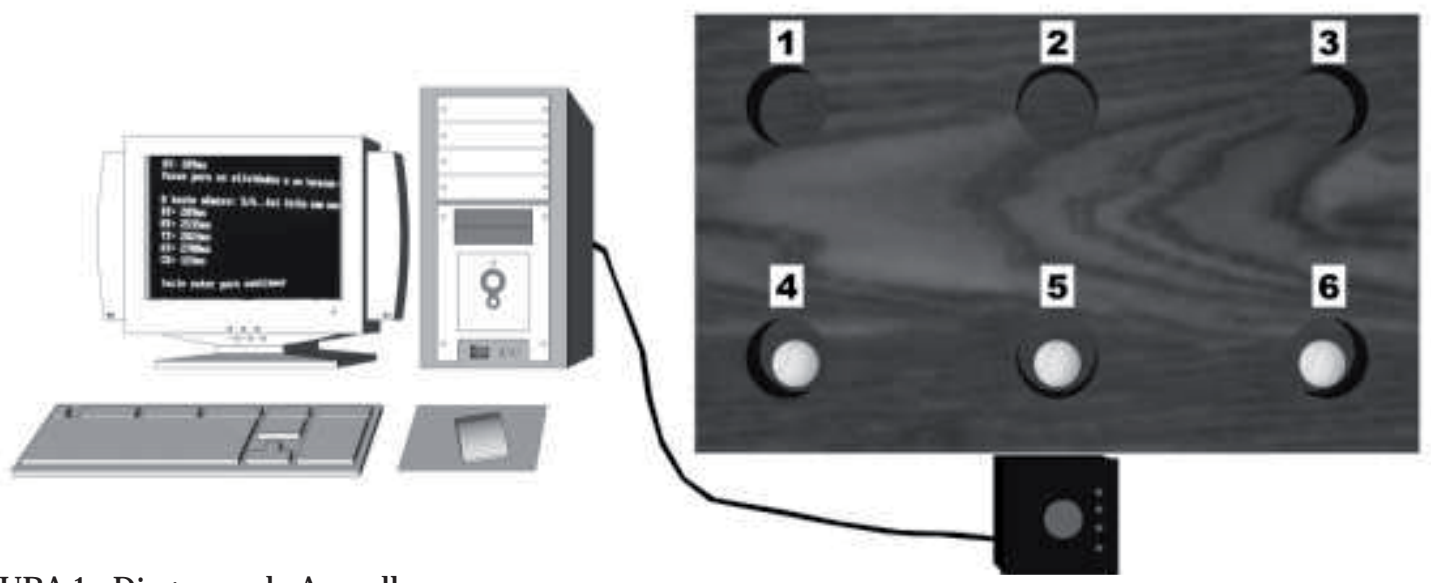

FIGURA 1 - Diagrama do Aparelho.

\section{Delineamento experimental}

Nove grupos experimentais foram compostos de forma parcialmente aleatória, de modo a apresentar uma distribuição similar entre ambos os sexos, que resultou em seis mulheres e quatro homens em cada grupo. Foram formados os grupos considerando três condições de intervalo intertentativas (QUADRO 1): três grupos com intervalo intertentativas de quatro segundos - G1 com intervalo pré-CR de quatro segundos ( $4 \mathrm{~s})$, sem pós-CR $(0 \mathrm{~s})$; $\mathrm{G} 2 \mathrm{sem}$ pré-CR $(0 \mathrm{~s})$, pós-CR de quatro segundos (4s); G3 com pré-CR de dois segundos (2s), pós-CR de dois segundos ( $2 \mathrm{~s})$; três grupos com intervalo intertentativas de oito segundos G4 com pré-CR de oito segundos $(8 \mathrm{~s})$, sem pós-CR $(0$ s); G5 sem pré-CR (0 s), pós-CR de oito segundos (8 s); G6 com pré-CR de quatro segundos (4 s), pós-CR de quatro segundos (4 s); e três grupos com intervalo intertentativas de 16 segundos - G7 com pré-CR 16 segundos (16 s), sem pós-CR (0 s); G8 sem pré-CR (0 s), pós-CR de16 segundos (16 s); G9 com pré-CR de oito segundos (8s), pós-CR de oito segundos (8).

$\mathrm{O}$ estudo apresentou fase de aquisição e testes de transferência. $\mathrm{O}$ experimento teve a duração de dois dias. No primeiro dia, foi realizada a fase de aquisição e, logo após, o teste de transferência imediata. No segundo dia, com intervalo aproximado de 24 horas, foi realizado o teste de transferência atrasada. $\mathrm{Na}$ fase de aquisição, os sujeitos praticaram 30 tentativas da tarefa de posicionamento manual, a qual consistia em transportar três bolas de tênis entre seis recipientes na ordem (4-2/5-3/6-1) no tempo alvo de $3000 \mathrm{~ms}$. Na fase de aquisição o CR verbal foi fornecido em magnitude e direção do erro, com frequência relativa de $100 \%$, diferenciando apenas o momento de seu fornecimento, conforme o delineamento experimental. Dez minutos após o término da fase de aquisição, foi aplicado o teste de transferência imediata e após 24 horas foi realizado o teste de transferência atrasada. Foram realizadas 15 tentativas de prática em cada teste e foram alterados, em ambos, o sequenciamento (6-1/5-3/42) e o tempo alvo ( $4000 \mathrm{~ms}$ ), além da ausência $\mathrm{CR}$. Nos testes, cada bloco de cinco tentativas possuía intervalo intertentativas de quatro, oito e 16 segundos respectivamente, com a finalidade de não propiciar vantagem para um grupo especifico, realizado sempre na mesma ordem. O número de tentativas e tempo alvo da fase de aquisição e testes, sequenciamento e intervalos entre a fases do experimento foram determinados por meio de estudo piloto.

QUADRO1- Delineamento experimental do estudo.

\begin{tabular}{lccc}
\hline Grupos & $\begin{array}{c}\text { Intervalo } \\
\text { Pré-CR }\end{array}$ & $\begin{array}{c}\text { Intervalo } \\
\text { Pós-CR }\end{array}$ & $\begin{array}{c}\text { Intervalo } \\
\text { Intertentivas }\end{array}$ \\
\hline G1 & $4 \mathrm{~s}$ & - & $4 \mathrm{~s}$ \\
G2 & - & $4 \mathrm{~s}$ & $4 \mathrm{~s}$ \\
G3 & $2 \mathrm{~s}$ & $2 \mathrm{~s}$ & $4 \mathrm{~s}$ \\
G4 & $8 \mathrm{~s}$ & - & $8 \mathrm{~s}$ \\
G5 & - & $8 \mathrm{~s}$ & $8 \mathrm{~s}$ \\
G6 & $4 \mathrm{~s}$ & $4 \mathrm{~s}$ & $8 \mathrm{~s}$ \\
G7 & $16 \mathrm{~s}$ & - & $16 \mathrm{~s}$ \\
G8 & - & $16 \mathrm{~s}$ & $16 \mathrm{~s}$ \\
G9 & $8 \mathrm{~s}$ & $8 \mathrm{~s}$ & $16 \mathrm{~s}$ \\
\hline
\end{tabular}




\section{Procedimentos}

A coleta de dados foi realizada de forma individual em sala apropriada, com temperatura e luminosidade controladas. Foi solicitado que os sujeitos se posicionassem em frente à plataforma onde receberam instrução verbal e demonstração consecutivamente por três vezes acerca da tarefa e da forma de fornecimento de CR. Após o sinal "prepara" (fornecido pelo experimentador), o sujeito deveria pressionar e sustentar a chave de respostas com a mão preferida e, após o estímulo

\section{Resultados}

Os dados foram organizados em blocos de cinco tentativas e os resultados foram analisados em relação ao erro absoluto e desvio padrão do erro absoluto na fase de aquisição e nos testes de transferência imediata (TI) e transferência atrasada (TA).

\section{Erro absoluto}

Uma Anova "two-way" (nove grupos x seis blocos) com medidas repetidas no segundo fator foi realizada na fase de aquisição e não detectou diferença significante entre grupos $[\mathrm{F}(8,81)=0,52 ; \mathrm{p}=0,83]$ ou interação entre grupos e blocos $[\mathrm{F}(40,405)=0,9$; $\mathrm{p}=0,64]$. Foi encontrada diferença significativa entre blocos $[F(5,405)=89,12 ; p<0,0001]$. O teste LSD detectou que o $1^{\circ}$ bloco apresentou maior erro que os demais blocos de tentativas ( $\mathrm{p}<0,0001)$ e que o $2^{\circ}$ bloco apresentou maior erro que o $5^{\circ}$ e $6^{\circ}$ blocos de tentativas $(\mathrm{p}<0,005)$ (FIGURA 2).

Uma Anova "two-way" (nove grupos x três blocos) com medidas repetidas no segundo fator foi realizada para o teste de transferência imediata e detectou diferença significativa entre grupos $[\mathrm{F}(8,81)=2,13$; $\mathrm{p}=0,042]$. O teste LSD registrou que o grupo G1 apresentou menor erro que os grupos G7, G8 e G9 ( $<<0,05)$; o grupo G9 apresentou maior erro que os grupos G1, G2, G3, G5 e G6 (p < 0,05). Não foi encontrada diferença significante no fator blocos $[F(2,162)=0,41 ; \mathrm{p}=0,67]$ ou interação entre grupos e blocos $[\mathrm{F}(16,162)=1,52 ; \mathrm{p}=0,1]$.

Outra Anova "two-way" (nove grupos x três blocos) com medidas repetidas no segundo fator foi aplicada para o teste de transferência atrasada e não detectou diferença significante no fator grupos $[\mathrm{F}(8,81)=1,3 ; \mathrm{p}=0,25]$, blocos $[\mathrm{F}(2,162)=$ visual (o acendimento dos diodos), a chave deveria ser liberada, iniciando o transporte das bolas de tênis, na ordem e tempo alvo pré-definidos. Após o transporte, a chave deveria ser novamente pressionada caracterizando o fim da tarefa. O CR foi então fornecido em magnitude e direção aos sujeitos conforme delineamento experimental.

Nos testes de transferência imediata e atrasada, o procedimento de utilização do aparelho foi o mesmo utilizado na fase de aquisição. Todavia houve a alteração do sequenciamento e do tempo alvo, e ausência de fornecimento de CR.
$0,27 ; \mathrm{p}=0,76]$ ou interação entre grupos e blocos $[\mathrm{F}(16,162)=0,91 ; \mathrm{p}=0,56]$.

\section{Desvio padrão do erro absoluto}

Uma Anova "two-way" (nove grupos x seis blocos) com medidas repetidas no segundo fator não detectou diferença significante entre grupos $[F(8,81)=1,5$; $\mathrm{p}=0,17]$. Encontrou-se diferença significativa no fator blocos $[F(5,405)=54,6 ; p<0,0001]$, e o teste LSD registrou maior variabilidade do primeiro em relação aos demais blocos de tentativas $(\mathrm{p}<0,001)$. Também foi encontrada interação significativa entre grupos e blocos $[\mathrm{F}(40,405)=1,42 ; \mathrm{p}=0,05]$. O teste LSD apontou maior variabilidade do $1^{\circ}$ bloco de tentativas do G8 sobre o primeiro bloco de tentativas dos demais grupos $(\mathrm{p}<0,05)$. Ainda, o $1^{\circ}$ bloco de tentativas do G3 apresentou menor variabilidade do que o $1^{\circ}$ bloco de tentativas dos grupos G1, G4, G5 e G6 (p < 0,05) (FIGURA 3).

Uma Anova "two-way" (nove grupos $\mathrm{x}$ três blocos) com medidas repetidas no segundo fator foi aplicada para o teste de transferência imediata e não verificou diferença significante entre grupos $[\mathrm{F}(8,81)=0,77 ; \mathrm{p}$ $=0,63]$ ou interação entre grupos e blocos $[F(16,162)$ $=0,78 ; p=0,7]$. Foi encontrada diferença significativa entre blocos $[\mathrm{F}(2,162)=11,31 ; \mathrm{p}<0,0001]$ e o teste LSD indicou maior variabilidade do primeiro sobre os demais blocos de tentativas do teste $(\mathrm{p}<0,001)$.

Outra Anova "two-way" (nove grupos x três blocos) com medidas repetidas no segundo fator foi aplicada para o teste de transferência atrasada e não encontrou diferença significante entre grupos $[\mathrm{F}(8,81)=0,83 ; \mathrm{p}=$ $0,58]$, blocos $[\mathrm{F}(2,162)=0,15 ; \mathrm{p}=0,86]$ ou interação entre grupos e blocos $[\mathrm{F}(16,162)=1,28 ; \mathrm{p}=0,21]$. 


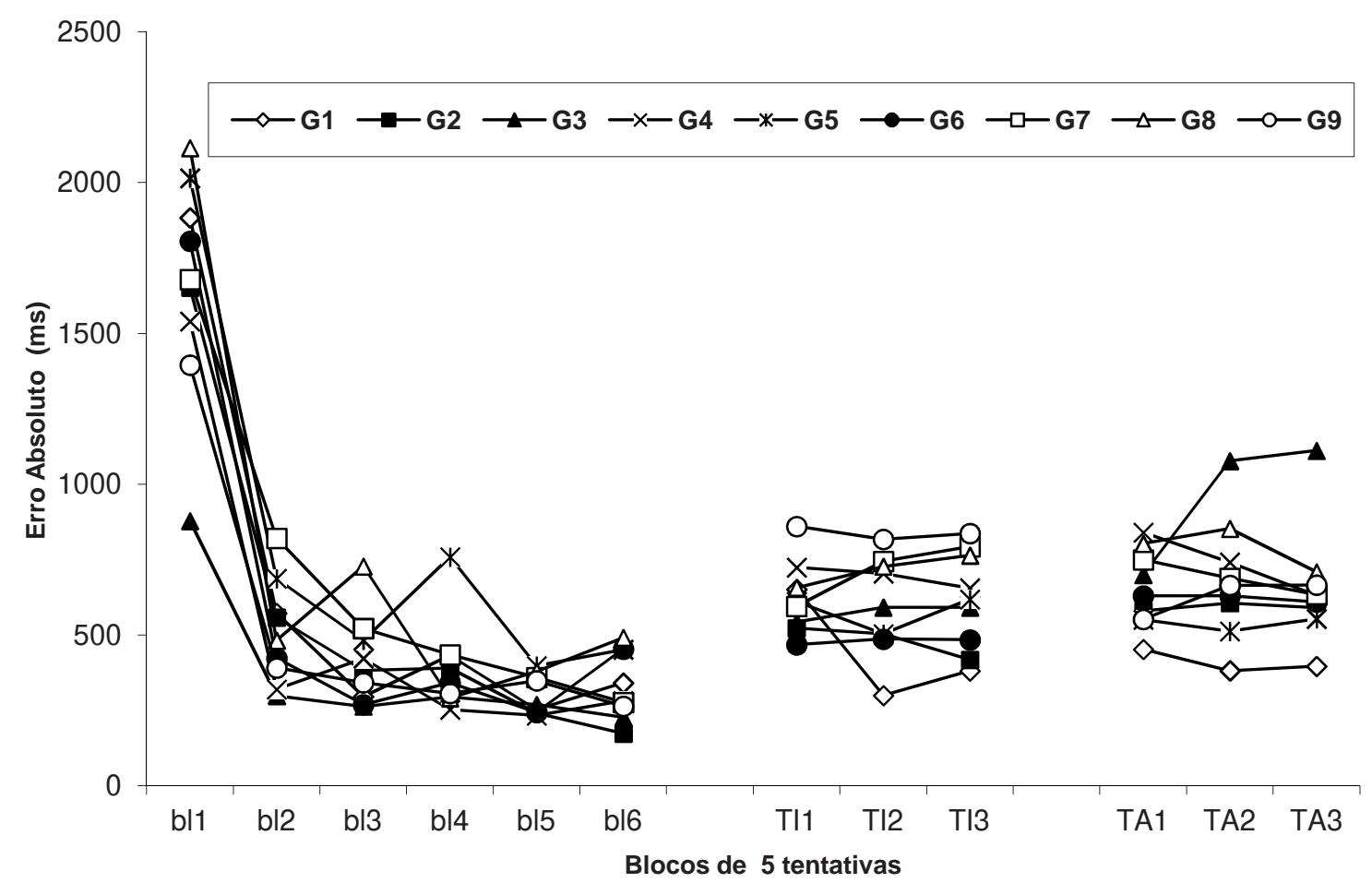

FIGURA 2 - Média do erro absoluto dos nove grupos experimentais na fase de aquisição e testes em blocos de cinco tentativas.

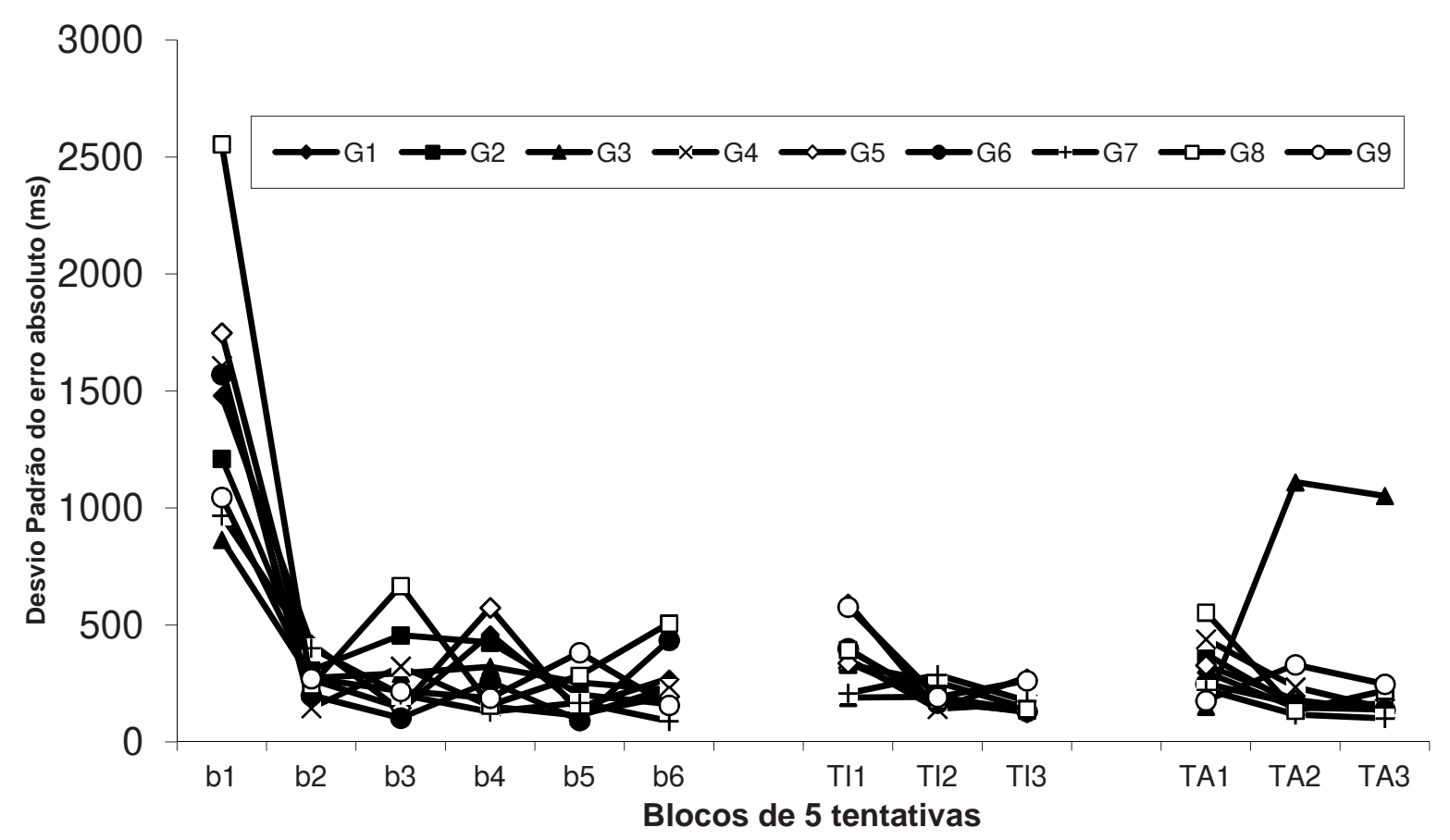

FIGURA 3 - Média do desvio padrão do erro absoluto dos nove grupos experimentais na fase deaquisição etestes em blocos de cinco tentativas. 


\section{Discussão}

O objetivo do presente estudo foi verificar os efeitos dos intervalos de tempo de apresentação de conhecimento de resultados na aquisição de habilidades motoras. De forma geral, os principais achados mostraram superioridade do grupo G1 sobre G7, G8 e G9, como inferioridade do grupo G9 em relação aos grupos G1, G2, G3, G5 e G6.

O desempenho dos grupos na fase de aquisição mostrou níveis similares de precisão e consistência, não importando presença, extensão ou ausência de qualquer dos intervalos de tempo. Os achados corroboram estudos prévios, que não detectaram influência da interação dos intervalos de tempo no desempenho de uma habilidade motora simples (DenY, Allard, HaLl \& ROKEACH, 1960) ou mesmo que sugeriram que tais diferenças não seriam observadas na fase de aquisição (SALMONI, SCHMidt \& Walter, 1984; Simmons \& SNyder, 1983). Apesar disso, é difícil generalizar esses achados, devido a alguns motivos, tais como a inconsistência dos resultados nas pesquisas sobre os intervalos de tempo de apresentação de CR, a utilização de diferentes tipos de tarefas e a ausência de desenho experimental capaz de mensurar efeitos duradouros de aprendizagem com uso de testes. Quanto ao teste de transferência imediata, o grupo G1, que tinha um intervalo intertentativas menor (quatro segundos), apresentou melhor desempenho quando comparado aos grupos G7, G8 e G9 que possuíam os maiores intervalos intertentativas (16 segundos). Além disso, o grupo G9 apresentou pior desempenho que os grupos G1, G2, G3, G5 e G6 com intervalos intertentativas menores (quatro e oito segundos). Esses resultados estão em conformidade com proposiçóes anteriores, que sugeriram maior efetividade de intervalos mais curtos na aquisição de habilidades motoras (KOEGEL, DUNLAP \& DYER, 1980; SAlmoni, Schmidt \& Walter, 1984).

A capacidade de processamento do "feedback" depende de fatores como complexidade da tarefa e da fase de aprendizagem em que se encontra o indivíduo. Destes fatores, aquele que se aproxima ao presente estudo é a fase de aprendizagem, pois a tarefa utilizada não é considerada como complexa. Assim, no início da aprendizagem, os indivíduos têm dificuldades para interpretar os estímulos que recebem devido à dificuldade de atribuir significado quando se trata da informação proprioceptiva, por exemplo. $\mathrm{Na}$ memória de curto prazo são realizadas as operações de comparação dos estímulos recebidos com a informação acumulada na memória de longo prazo, o que permite destacar o aspecto relevante de uma determinada situação (GODINHO \& MENDES, 1996).

A principal fundamentação sobre os efeitos positivos do intervalo pré-CR na aprendizagem motora está no tempo necessário para processar o "feedback" intrínseco e proporcionando a formação de um mecanismo de detecção e correção de erros. Tal comportamento é verificado quando grupos com "feedback" imediato apresentam bons desempenhos na fase de aquisição, devido à presença do $\mathrm{CR}$, mas um desempenho inferior nos testes de retenção e transferência (McGuigan, 1959; PALHARES et al., 2006; SWINNEN et al., 1990). Sugere-se então que o pré-CR não deve apresentar intervalos muito curtos porque impossibilitaria o indivíduo ter tempo suficiente para utilizar as informaçôes advindas do "feedback" intrínseco, o que poderia influenciar negativamente o desempenho (GodinHo \& Mendes, 1996; SwINNEN et al., 1990). Por outro lado, intervalos muito longos podem causar diminuição da motivação, da atenção e esquecimento. Dessa forma, as informações sobre o movimento executado se perderiam na memória de trabalho e o CR perderia o papel de referência na correção do erro, inibindo a melhora da tentativa seguinte, acarretando assim prejuízo na aprendizagem motora (SimMONS \& SNYDER, 1983).

No presente estudo, a combinação entre os diferentes processos cognitivos pode ser uma das explicaçōes acerca do pior desempenho dos grupos com intervalo intertentativas de 16 segundos. $\mathrm{O}$ tempo provavelmente interfere no processamento de informaçôes, principalmente quando se envolve a atenção, percepção e memória. Ao analisar o CR fornecido, é necessário atenção a esta nova informação, percepção para fazer as comparaçóes e reconhecimento de valor, obviamente associado à memória que armazenou os parâmetros do movimento realizado recentemente (AdAMS, 1971; MarTENIUK, 1976). GOdinHO e MenDES (1996) ressaltaram que operaçôes de comparação são realizadas na memória de curto prazo.

A memória se caracteriza pelo armazenamento e recuperação de eventos passados e está intimamente ligada à atenção, percepção e aos sistemas de processamento de informação (CRAIK, 1979; Norman, 1968). Intervalos de tempo muito longos proporcionam problemas no processo de codificação da informação a ser recuperada, utilizando-se como referência os intervalos curtos comparados a longos. Essa explicação corrobora as explanaçôes de Marteniuk (1975), em que os estímulos são 
apresentados em série ao indivíduo que atende a cada item seletivamente. Assim, uma sequência muito extensa extrapola a capacidade de reserva proporcionando o esquecimento dos itens apresentados posteriormente. Neste caso, um mecanismo de detecção e correção de erros poderia não ser formado, pois as informaçōes pertinentes para a formação da resposta não seriam acessadas com o máximo de certeza, uma vez que foram esquecidas, interferindo no processo de controle e de pesquisa da memória de curto prazo, produzindo um mecanismo de recuperação ineficiente (AtKInSON \& SHIFFrin, 1971).

A memória de curto prazo age no processo de aprendizagem motora imediatamente após o desempenho da habilidade motora. Essa visão está apoiada na formação de um mecanismo de solução de problemas que se baseia no "feedback" proprioceptivo, bem como as informaçôes auditiva e visual. Esse mecanismo está presente na memória de curto prazo e utiliza atenção e memória para a recuperação da informação. Ainda, a memória de curto prazo representa um sistema no qual a informação rapidamente se perde na ausência da manutenção da atenção, o que pode levar a um possível esquecimento (MARTENIUK, 1975). No presente estudo, os intervalos mais longos podem ter diminuído os níveis de atenção, interferindo nos mecanismos perceptivos, e assim ocasionaram algum tipo de ruído, levando à perda da informação nos grupos com intervalos intertentativas de 16 segundos, que apresentaram resultados inferiores a um grupo com intervalo intertentativas de quatro segundos. Os resultados do presente estudo sugerem a importância dos intervalos de apresentação de CR, especialmente os intervalos intertentativas e pré-CR, que parecem ser essenciais na aprendizagem de habilidades motoras. Em relação ao intervalo pós-CR, este foi restringido pela extensão do intervalo intertentativas.

O presente estudo apresenta evidências contrárias a estudos anteriores (BOURNE \& BUNDERSON, 1963; Ramella, 1983, Simmons \& SNyder, 1983), uma vez que tais indicaçôes foram propostas quando somente uma variável independente foi investigada (intervalo pré-CR ou intervalo pós-CR). Ao se considerar diferentes intervalos analisados em conjunto, verifica-se que tempos mais longos do intervalo préCR (Palhares et al., 2006; Swinnen et al., 1990) com os tempos mais longos do intervalo pós-CR (Gallagher \& Thomas, 1980; Magill, 1988) podem resultar em intervalos intertentativas que deterioram a aprendizagem motora. Assim sendo, tais citações precisam ser analisadas considerando também o intervalo intertentativas.

Em conclusão, os resultados encontrados sugerem que os intervalos intertentativas mais curtos, com ênfase ao pré-CR, seriam capazes de influenciar positivamente a aquisição de habilidades motoras em detrimento de intervalos intertentativas mais longos, como a literatura sugere. Cabe questionar se estes achados serão reproduzidos em tarefas mais complexas tais como as observadas em situação real de ensino-aprendizagem.

\section{Abstract}

Effects of temporal locus of knowledge of results (KR) in motor skills acquisition

The temporal locus of the Knowledge of Results (KR) is composed by three intervals: pre KR, post-KR, and inter trial interval. A problem regarding this topic is that the manipulation of one interval implies in changing the others, suggesting that the intervals may be combined for analysis. The present study investigated the effects of temporal locus of the KR in the motor skills acquisition. A manual positioning task consisting of the transport of three tennis balls between six containers in a specific sequence and time was used. The apparatus was comprised of a platform with six containers and a central controller connected to a computer. An acquisition phase of 30 trials using the 4-2/5-3/6-1 sequence and a 3,000 msec target time was allowed. The immediate transfer tests and the delayed transfer tests were performed ten minutes and twenty four hours after the completion of the acquisition phase, respectively. Both tests were preceded by 15 practice trials in a previously unknown movement sequence (6-1/5-3/4-2) and target time $(4,000$ msec.) without any KR. Ninety undergraduate students were allocated into nine groups $(n=10)$. Three groups had a four-second interval between trials; three groups had an eightsecond intertrial interval; the reaming three groups had a sixteen-second intertrial interval. All groups had distinct pre and post-KR intervals. The results showed that smaller intertrial intervals resulted in 
better testing performance. It suggests that the time for information processing should not be long, as it increases both the attention and memory demands.

UNITERMS: Feedback; Knowledge of results; KR Delay; Intertrial interval.

\section{Referências}

ADAMS, J.A. A closed-loop theory of motor learning. Journal of Motor Behavior, Washington, v.3, p.111-49, 1971. ATKINSON, R.C.; SHIFFRIN, R.M. The control short-term memory. Scientific American, New York, v.2, p.82-90, 1971. BECKER, P.W. Are simple line-length estimation tasks productive for examining temporal locus of knowledge of results? Perceptual and Motor Skills, Missoula, v.91, p.801-2, 2000.

BECKER, P.W.; MUSSINA, C.M.; PERSONS, R.; W. Intertrial interval delay of knowledge of results, and motor performance. Perceptual and Motor Skills, Missoula,v.17, p.559-63, 1963.

BILODEAU, E.A.; BILODEAU, I.M. Variation of temporal intervals among critical events in five studies of knowledge of results. Journal of Experimental Psychology, Washington, v.55, p.603-12, 1958.

BOURNE, L.E.; BUNDERSON, C.V. Effects of delay of information feedback and length of postfeedback interval on concept identification. Journal of Experimental Psychology, Washington, v.65, p.1-5, 1963.

CARNAHAN, H.; HALL, C.; LEE, T.H. Delayed visual feedback while learning to track a moving target. Research Quarterly for Exercise and Sport, Washington, v.67, p.416-23, 1996.

CRAIK, F.I.M. Human memory. Annual Review Psychology, Palo Alto, v.30, p.63-102, 1979.

DENNY, M.R.; ALLARD, M.; HALL, E.; ROKEACH, M. Supllementary report: delay of knowledge of results, knowledge of task, and intertrial interval. Journal of Experimental Psychology, Washington, v.60, p.327, 1960.

GALLAGHER, J.D.; THOMAS, J.R. Effects of varying post-KR intervals upon children's motor performance. Journal of Motor Behavior, Washington, v.12, p.41-56, 1980.

GODINHO, M. Informação de retorno e desenvolvimento motor. Ludens, Lisboa, v.12, p.59-61, 1992.

GODINHO, M.; MENDES, R. Aprendizagem motora: informação de retorno sobre o resultado. Lisboa: Ediçōes FMH, 1996. KOEGEL, R.L.; DUNLAP, G.; DYER, K. Intertrial interval duration and learning in autistic children. Journal Applied Behavior Analysis, Lawrence, v.13, p.91-99, 1980.

LIU, J.; GERVAIS, P.D.; CROSTON, A.L. The effect of subjective estimation of performance outcome during the KRdelay interval on the acquisition and retention of a motor skill. Research Quarterly for Exercise and Sport, Washington, supplement, p.45-51, 2001.

LIU, J.; WRISBERG, C.A. The effect of knowledge of results delay and the subjective estimation of movement form on the acquisition and retention of a motor skill. Research Quarterly for Exercise and Sport, Washington, v.68, p.145-51, 1997. MAGILL, R.A. Activity during the post-knowledge of result interval can benefit motor skill learning. In: MEIJER, O.G.; ROTH, K. (Eds.). Complex motor behavior: 'the' motor-action controversy, Amsterdam: Elsevier, 1988. p.231-46.

MAGILL, R.A. The influence of augmented feedback on skill learning depends on characteristics of the skill and the learner. Quest, Champaign, v.46, p.314-27, 1994.

. The processing of knowledge of results information for a serial-motor task. Journal of Motor Behavior, Washington, v.9, p.113-118, 1977.

MARTENIUK, R.G. Information processing, channel capacity, learning stages and the acquisition of motor skill. In: WHITING, H.T.A. (Ed.). Readings in human performance. London: Lepus, 1975. p.5-33.

. Information processing in motor skills. Waterloo: Holt, Rinehart and Winston, 1976.

McGUIGAN, F.J. Delay of knowledge of results: a problem in design. Psychological Reports, Missoula, v.5, p.241-3, 1959. NORMAN, D.A. Toward a theory of memory and attention. Psychological Review, Pittsburgh, v.6, p.522-536, 1968. PALHARES, L.R.; LAGE, G.M.; VIEIRA, M.M.; UGRINOWITSCH, H.; BENDA, R.N. KR-delay interval effects in acquisition of positioning tasks in different compatibility levels. Journal of Human Movement Studies, Edimburg, v.51, p.47-61, 2006.

RAMELLA, R.J. Processing, knowledge of results, and a multi-dimensional task. Perceptual and Motor Skills, Missoula, v.57, p.43-48, 1983.

SALMONI, A.W.; SCHMIDT, R.A.; WALTER, C.B. Knowledge of results and motor learning: a review and critical reappraisal. Psychological Bulletin, Washington, v.95, p.355-86, 1984. 
SCHMIDT, R.A. A schema theory of discrete motor skill learning. Psychological Review, Washington, v.82, p.225-60, 1975.

Motor control and learning: a behavioral emphasis. 2nd. ed. Champaign: Human Kinetics, 1988.

SIMMONS, R.W.; SNYDER, R.J. Variation of temporal locus of knowledge of results: effects on motor performance of a simple task. Perceptual and Motor Skills, Missoula, v.56, p.399-404, 1983.

SWINNEN, S.P. Information feedback for motor skill learning: a review. In: ZELAZNIK, H.N. (Ed.). Advances in motor learning and control. Champaign: Human Kinetics, 1996. p. 37-66.

SWINNEN, S.P.; SCHMIDT, R.A.; NICHOLSON, D.E.; SHAPIRO, D.C. Information feedback for skill acquisition: instantaneous knowledge of results degrades learning. Journal of Experimental Psychology: Learning, Memory and Cognition, Washington, v.16, p.706-16, 1990.

TRAVLOS, A.K. Re-examining the temporal locus of knowledge of results (KR): a self-paced approach to learning. Perceptual and Motor Skills, Missoula, v.89, p.1073-87, 1999.

TRAVLOS, A.K.; PRATT, J. The temporal locus of knowledge of results: a meta-analytic review. Perceptual and Motor Skills, Missoula, v.80, p.3-14, 1995.

VIEIRA, M.M.; ENNES, F.C.M.; LAGE, G.M.; PALHARES, L.R.; UGRINOWITSCH, H.; BENDA, R.N. Os efeitos do intervalo pós-conhecimento de resultados na aquisição do arremesso da bocha. Revista Portuguesa de Ciências do Desporto, Porto, v.6, p.50-4, 2006.

WEINBERG, D.R.; GUY, D.E.; TUPPER, R.W. Variations of post feedback interval in simple motor learning. Journal of Experimental Psychology, Washington, v.67, p.9899, 1964.

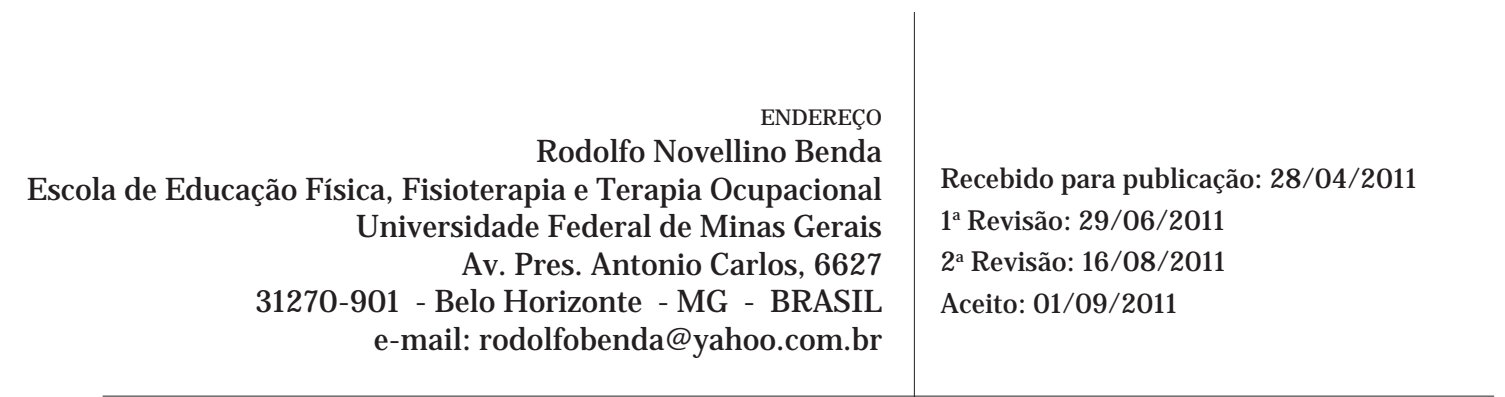

